\title{
Caracterización de los hallazgos histopatológicos de tumores hipofisiarios y de la región selar en dos centros en Bucaramanga, Santander, entre 1992 y 2018
}

Characterization of histopathological findings of pituitary tumors and the sellar region of two centers in Bucaramanga, Santander, between 1992 and 2018

\author{
Juan Guillermo Sarmiento ${ }^{1}$, María Paula Sarmiento ${ }^{2}$, Laura Catalina Aguirre ${ }^{2}$, Ludwing \\ Joel Valero ${ }^{2 *}$, Diana Katherine Saiz ${ }^{2 *}$, Karoll Dayanna Aparicio ${ }^{2 *}$, Jorge Luis Sanabria ${ }^{2 *}$, Silvia \\ Nathalia Gutiérrez ${ }^{2 *}$, María Emma García ${ }^{3}$, Isabel Cristina Bolívar ${ }^{3}$
}

${ }^{1}$ Grupo endocrinología, FOSCAL. Bucaramanga, Colombia.

${ }^{2}$ Facultad de Ciencias de la Salud, Universidad Autónoma de Bucaramanga. Bucaramanga, Colombia.

${ }^{3}$ Grupo patología, FOSCAL. Bucaramanga, Colombia.

*Contribución en igual medida al desarrollo del estudio Institución: Fundación Oftalmológica de Santander - Clínica Carlos Ardila Lulle (FOSCAL), Unidad de Endocrinología de FOSCAL Internacional, Bucaramanga, Santander. Institución de práctica de la Universidad Autónoma de Bucaramanga.

Correspondencia: Juan Guillermo Sarmiento Ramón Correo electrónico: jgsarmientor@gmail.com.

Fecha de recepción: 7/10/2018

Fecha de aceptación: 21/11/2018

\section{Resumen}

Introducción: La hipófisis y la región selar pueden afectarse por una amplia variedad de lesiones de diferente etiopatogenia. En Colombia existen pocos datos que muestren la distribución y las características histopatológicas de dichas lesiones.

Objetivo: Describir las características histopatológicas de las lesiones hipofisarias y de la región selar en dos instituciones de la región de Santander, Colombia.

Materiales y métodos: Estudio descriptivo y retrospectivo. Se revisaron patologías de pacientes de toda edad con diagnóstico de patología selar, supraselar o paraselar, llevados a cirugía realizada en dos centros de referencia de la región.

Resultados: Se revisaron 111 estudios patológicos, la lesión más frecuente fue el adenoma hipofisario $(66,6 \%)$, seguido del craneofaringioma $(19,8 \%)$ y del meningioma $(2,5 \%)$. En población pediátrica, el craneofaringioma fue la lesión predominante (75\%). Dentro de los adenomas predominaron los de células nulas y gonadotropos, la mayoría mostraron una expresión baja de Ki-67. La descripción heterogénea de los hallazgos de la citoqueratina CAM 5.2 y microscopia electrónica impidió su clasificación según dichos hallazgos. La variante más frecuente del craneofaringioma, tanto en adultos como en niños, fue la adamantinomatosa.

Conclusión: La distribución de la patología hipofisaria y selar en esta serie es similar a lo reportado en la literatura, llamando la atención un predominio del craneofaringioma como la segunda lesión más frecuente después de los adenomas hipofisarios. Se requiere una mayor uniformidad y difusión en los criterios histopatológicos de la clasificación de los adenomas para una interpretación clínica más apropiada y un uso óptimo de los recursos.

Palabras clave: enfermedades de la hipófisis, hipófisis, silla turca, adenoma, patología, patología quirúrgica, craneofaringioma, Colombia.

\section{Summary}

Introduction: The pituitary gland and the sellar region can be affected by a wide variety of lesions that originate via different pathophysiological mechanisms. In Colombia, there is scarce data reflecting the distribution and histopathological characteristics of these lesions.

Objective: To describe the histopathological characteristics of the pituitary gland and sellar region lesions in two institutions of the Santander region in Colombia.

Materials and methods: Descriptive and retrospective study. Pathology reports from patients of all ages from two reference centers in the region with a diagnosis of sellar, suprasellar or parasellar disease that underwent surgical resection were included in this study. 
Results: 113 pathology reports were reviewed. The lesions most frequently identified were pituitary adenomas (66.6\%), followed by craniopharyngiomas (19.8\%) and meningiomas (2.5\%). In the pediatric population, craniopharyngiomas were the predominant lesions (75\%). Within the adenomas, those of null and gonadotropic cell origins were the most prevalent tumors and most of them showed a low expression of Ki-67. The inconsistent description of the presence of cytokeratin CAM 5.2 and of electron microscopy prevented the classification of the lesions based on these findings. The most frequently identified variant of craniopharyngiomas in both adults and children was the adamantinomatous variant.

Conclusion: The distribution of the pituitary and sellar lesions in this study was similar to that reported in the literature. Importantly, craniopharyngiomas were identified as the second most common lesions after pituitary adenomas. A greater degree of uniformity and widespread use of histopathological criteria of pituitary adenomas is required in order to attain a more appropriate clinical interpretation and an optimal use of these resources.

Key words: pituitary disease, pituitary gland, piuitary neoplasms, surgical pathology, craniopharyngiomas, Colombia.

\section{Introducción}

La hipófisis es un órgano esencial en la homeostasis de todos los sistemas del cuerpo, puede afectarse por patologías propias de la glándula o de la región selar, produciendo manifestaciones por déficit o exceso hormonal y compromiso de estructuras nerviosas adyacentes, principalmente visuales. Dentro de la patología que puede afectarla se debe considerar una amplia gama de diagnósticos diferenciales de carácter infeccioso, infiltrativo y tumoral, entre otros; siendo los adenomas los más frecuentes, representando hasta el 15\%-20\% de las neoplasias intracraneales ${ }^{(1,2,3)}$. En una revisión sistemática de estudio de autopsias e imágenes por resonancia magnética se encontró que la prevalencia poblacional de adenomas pituitarios fue del $16,7 \%$, mostrando que con frecuencia estas lesiones se encuentran de manera incidental ${ }^{(4,5)}$.

Varios estudios de diferentes centros del mundo han reportado la distribución de la patología hipofisaria y de la región selar ${ }^{(6,7)}$, siendo el registro alemán el de mayor tamaño con 4.122 casos recolectados en diferentes centros clínicos, la patología más prevalente fueron los adenomas $(84,6 \%)$, seguidos por otros tumores como craneofaringiomas $(3,2 \%)$, meningiomas $(0,94 \%)$ y con una prevalencia muy baja se distribuyeron el resto de casos entre: cordomas $(0,55 \%)$, carcinomas pituitarios $(0,12 \%)$, metástasis $(0,6 \%)$, lesiones quísticas no neoplásicas $(2,8 \%)$ y lesiones inflamatorias $(1,1 \%)^{(6)}$.

En Colombia existen pocos estudios que describan la distribución global de los hallazgos histopatológicos de las lesiones hipofisarias y la región selar. Teniendo en cuenta la amplia gama de diagnósticos diferenciales que pueden manifestarse en esta región y su impacto clínico, es esencial conocer el comportamiento de dichas lesiones en nuestro medio.

\section{Materiales y métodos}

Se llevó a cabo un estudio descriptivo y retrospectivo. Se revisaron los estudios patológicos de pacientes de toda edad con diagnóstico de patología selar, supraselar o paraselar que fueron llevados a cirugía realizada en FOSCAL o FOSCAL internacional, entre enero de 1992 y enero de 2018. Se excluyeron aquellos casos en los cuales no se encontraban todos los datos requeridos para el estudio en el registro.

Los casos potenciales eran identificados por la búsqueda en la base de datos de dos de los laboratorios de patología de las instituciones, usando los términos selar, supraselar, paraselar, hipófisis o pituitario. Se diseñó un instrumento para recopilar los datos sobre variables demográficas e histología de lesión. Se clasificaron en seis grupos: hipófisis normal, craneofaringioma, meningioma, hipofisitis, cordoma y adenoma. Este último grupo se describió según la inmunohistoquímica, clasificación de Ki-67\%, identificación de la expresión de P53 y presencia de hallazgos compatibles con apoplejía. Igualmente se obtuvo información de microscopía electrónica en el caso de los adenomas hipofisarios. Finalmente, se clasificaron según lo establecido por la Organización Mundial de la Salud (OMS) en 2017.

\section{Consideraciones éticas}

Se condujo bajo los criterios y parámetros establecidos por la Resolución 8430 de 1993. Este es un estudio observacional descriptivo, usa datos de registro médico e histopatología recolectada de una institución. No requiere aprobación de Comité de Ética.

\section{Análisis estadístico}

La captura de datos se realizó en una base de datos de Excel, posteriormente se importó en EPIINFO versión 7.2 para su análisis estadístico. Se describieron las características de los hallazgos según la naturaleza de las variables. Las variables cuantitativas, se analizaron por mediana y rango; mientras que las variables cualitativas se analizaron por proporciones.

\section{Resultados}

Se revisaron un total de 111 estudios patológicos, recolectados entre enero de 1992 y enero de 2018. La mediana de edad fue de 50 años, con un rango entre 3 y 82 años. Las mujeres representaron $62(55,9 \%)$ de los casos. La lesión más frecuentemente identificada fue adenoma con 74 casos $(66,6 \%)$, seguido del craneofaringioma con 22 casos $(19,8 \%)$ y meningioma con 5 casos $(2,5 \%)$, el resto de distribución se representa en la tabla 1 y la figura 1 . Al realizar la descripción de 
Tabla 1. Distribución general del tipo de lesión

\begin{tabular}{l|c|c}
\multicolumn{1}{c|}{ Lesión } & $\begin{array}{c}\text { Frecuencia } \\
\text { (n=11) }\end{array}$ & Porcentaje (\%) \\
\hline Adenoma & 74 & $66,6 \%$ \\
\hline Craneofaringioma & 22 & $19,8 \%$ \\
\hline Meningioma & 5 & $4,5 \%$ \\
\hline Tumor germinal & 1 & $0,9 \%$ \\
\hline Cordoma & 4 & $3,6 \%$ \\
\hline Metástasis & 1 & $0,9 \%$ \\
\hline Hipófisis sana & 2 & $1,8 \%$ \\
\hline $\begin{array}{l}\text { Carcinoma de } \\
\text { hipófisis }\end{array}$ & 1 & $0,9 \%$ \\
\hline $\begin{array}{l}\text { Granulomatosis } \\
\text { con poliangeítis }\end{array}$ & 1 & $0,9 \%$ \\
\hline
\end{tabular}

Figura 1. Distribución general del tipo de lesión

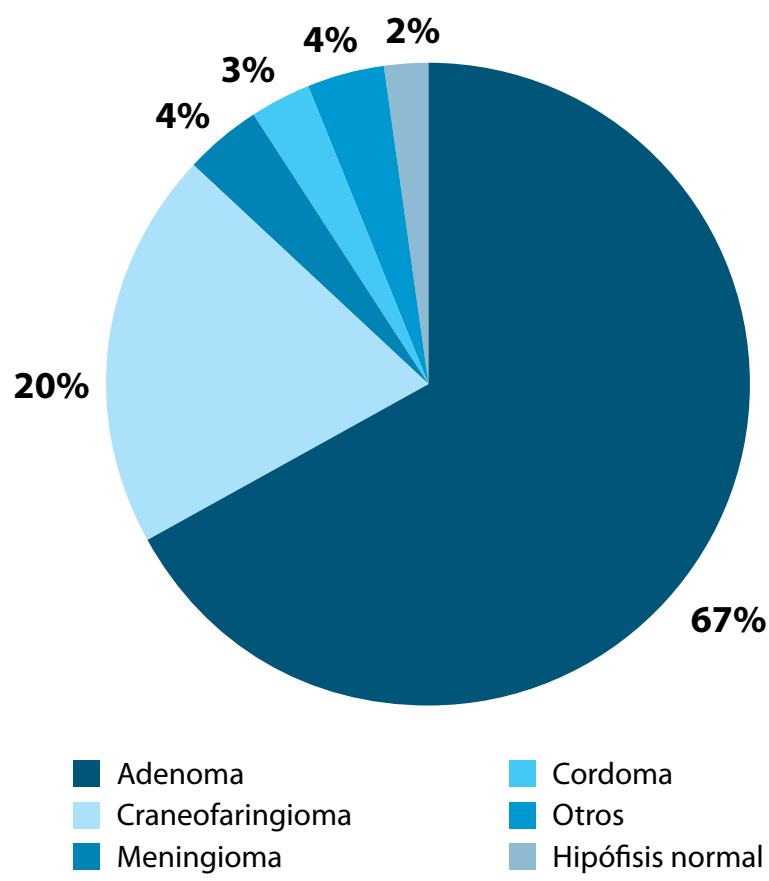

las lesiones por sexo, todas presentaron distribución similar, a excepción de meningioma en el cual todos los casos se presentaron en mujeres. Finalmente, al caracterizar la lesión por edad, los mayores de 18 años presentaban como lesión más frecuente adenoma con 72 casos $(72,7 \%)$ y en los menores de 18 años el craneofaringioma fue el más representativo con 9 casos (75\%), (figura 2). Dentro de los hallazgos pediátricos, el adenoma ocupó el segundo lugar, con un $16,6 \%$ y, finalmente, el cordoma con $8,3 \%$ de los casos.

Al identificar las características de los 74 adenomas presentados, en 44 de los casos $(59,4 \%)$ se reportó inmunohis-
Figura 2. Distribución del tipo de lesión en población pediátrica

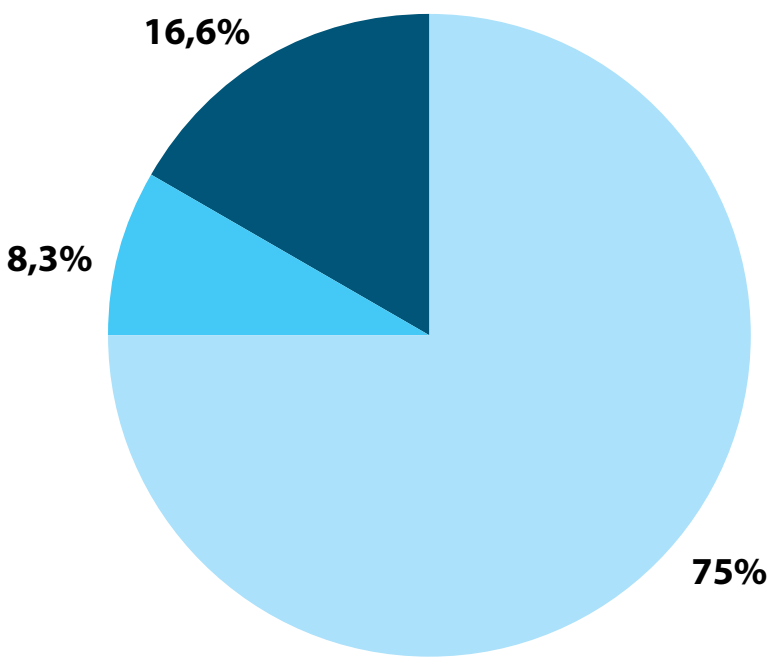

Adenoma Craneofaringioma

Cordoma

Figura 3. Clasificación inmunohistoquímica de los adenomas según el tipo de hormona expresada

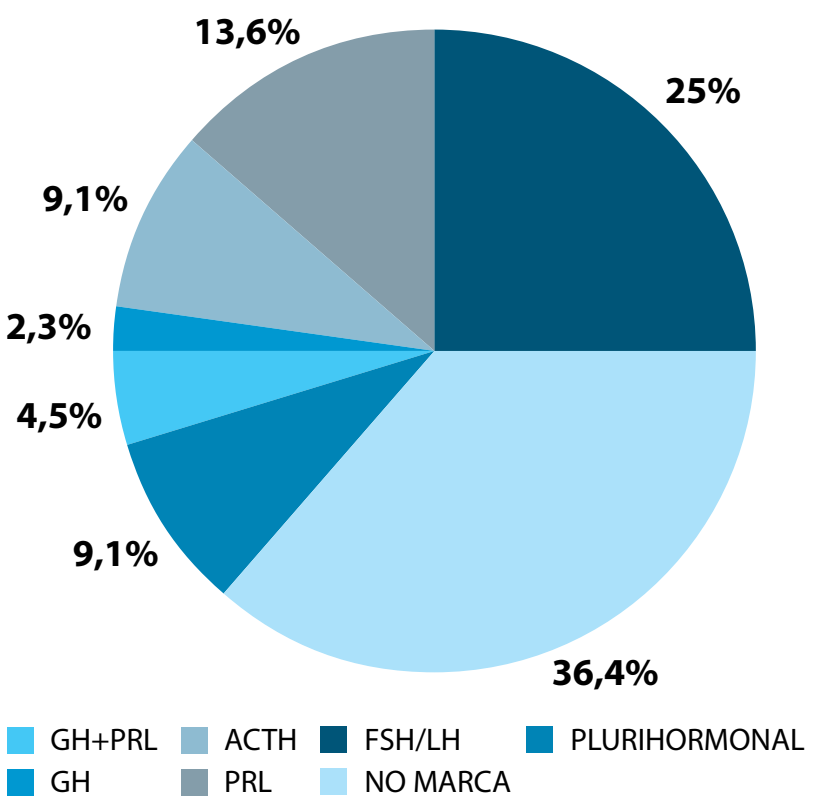

GH: hormona de crecimiento; PRL: prolactina; ACTH: hormona adrenocorticotropa; FSH: Hormona folículo estimulante; LH: hormona luteinizante

toquímica, lo cual permitió clasificar el tumor según el tipo de hormona expresada, siendo los que no expresaron (células nulas) y los gonadotropos los más frecuentes. En la figura 3 se representan estos hallazgos. Por otro lado, a $42(56,8 \%, \mathrm{n}=74)$ de los adenomas se les evaluó el porcentaje de expresión Ki67 ; con un promedio de $1,58 \%$ y 5 casos $(11,9 \%, n=42)$ pre- 
Tabla 2. Distribución de variante de craneofaringioma según edad

\begin{tabular}{l|c|c|c|c}
\hline \multirow{2}{*}{\multicolumn{1}{c|}{ Variante }} & \multicolumn{2}{c|}{ Frecuencia } & \multicolumn{2}{c}{ Porcentaje (\%) } \\
\cline { 2 - 5 } & $\begin{array}{c}\text { Menor o igual a 18 } \\
\text { años }(n=9)\end{array}$ & $\begin{array}{c}\text { Mayor a 18 años } \\
(\mathrm{n}=13)\end{array}$ & $\begin{array}{c}\text { Menor o igual a 18 } \\
\text { años }(\mathrm{n}=9)\end{array}$ & $\begin{array}{c}\text { Mayor a 18 años } \\
(\mathrm{n}=13)\end{array}$ \\
\hline Adamantinomatoso & 8 & 7 & $88,9 \%$ & $53,8 \%$ \\
\hline Papilar & 0 & 0 & - & - \\
\hline Sin variante & 1 & 6 & $11,1 \%$ & $46,1 \%$ \\
\hline
\end{tabular}

sentaron un porcentaje Ki- $67 \geq 3 \%$, considerándose proliferativo. A 31 casos $(41,8 \%, n=74)$ se les realizó P53, siendo todos los reportes negativos. Adicionalmente, a $16(21,6 \%, \mathrm{n}=74)$ casos se les evaluó microscopía electrónica y a 29 (39\%,n=74) casos CAM 5.2. Sin embargo, este hallazgo fue heterogéneo, lo cual no permitió una categorización de los datos. Finalmente, $8,1 \%$ de los adenomas presentaron cambios compatibles con apoplejía, siendo $50 \%$ isquémicos y $50 \%$ hemorrágicos.

Con respecto a los hallazgos de los craneofaringioma, la variante adamantinomatoso fue la más frecuente con $15(68,1 \%)$ casos. Al comparar los hallazgos de variante según la edad, los menores y mayores de 18 años presentaron como variante predominante la adamantinomatosa con $8(88,9 \%)$ casos y 7 $(53,8 \%)$ casos, respectivamente, (tabla 2$)$.

\section{Discusión}

En este estudio retrospectivo se evaluaron las características histopatológicas de las lesiones selares llevadas a cirugía en un centro de referencia de la región. En Colombia existen pocos estudios que describan los hallazgos histológicos de las lesiones hipofisarias y no existen hasta el momento estudios al respecto en la región de Santander. En general, los hallazgos encontrados son similares a los reportados en la literatura internacional $^{(6,7)}$; sin embargo, hay algunos datos llamativos que se deben analizar.

Respecto a la distribución global de las lesiones encontradas, como es bien conocido, la lesión predominante fue claramente el adenoma hipofisario, llamando la atención el predominio del craneofaringioma como la segunda lesión más frecuente (19\%), con una prevalencia más alta respecto a lo reportado en otras series $(<5 \%)^{(6,7)}$. Se encontraron de igual manera casos de meningioma y cordomas con una prevalencia por encima de lo esperado.

Dentro de los hallazgos inmunohistoquímicos de los adenomas, se encontró una distribución por el tipo de hormona expresada similar a lo reportado previamente, con un predominio de los adenomas silentes y gonadotropos ${ }^{(6,7,8)}$. Al tratarse de un estudio solo de muestras quirúrgicas, la prevalencia del prolactinoma cuya primera línea de manejo es médica y de algunos tumores sin impacto clínico probablemente queda subestimada y estos hallazgos no representan la distribución real de todos los adenomas encontrados en la práctica clínica. En general, el Ki-67 de los adenomas fue bajo, y la mayoría de los adenomas se caracterizaron como "no proliferativos" según la clasificación propuesta por el grupo francés de Trouillas ${ }^{(9)}$.

Es de resaltar que a pesar de que el 39\% de los adenomas tenían evaluación de citoqueratina CAM 5.2 y $21 \%$ de microscopía electrónica, su interpretación fue muy heterogénea y no se basó en un criterio unificado de reporte que permitiera establecer si el tumor en densa o dispersamente granular, dato que se ha demostrado en algunos subtipos de adenoma puede tener un valor pronóstico y orientar el manejo médico ${ }^{(10)}$. Con una adecuada interpretación de la inmunohistoquímica, en la mayoría de casos no es necesario, según las indicaciones de la OMS, realizar microscopía electrónica ${ }^{(11)}$. Por tanto, se requiere una mayor difusión entre los patólogos de los criterios de clasificación de tumores hipofisarios, recientemente publicados por la OMS en el 2017, para tener datos que se reporten de manera más homogénea, que faciliten las conductas clínicas y optimicen el uso de recursos.

Como se mencionó, el craneofarginoma fue la segunda lesión selar más frecuente, siendo la lesión predominante en población pediátrica. Dentro las variantes histológicas, fue llamativo que la variante adamantinomatosa fue la más frecuente en adultos, lo cual contrasta con los datos previamente conocidos que indican que la variante predominante en esta población es la papilar ${ }^{(12,13)}$. Se deberán corroborar estos datos con más estudios, dado que recientemente se ha encontrado que las variantes del craneofaringioma tienen un origen oncogénico diferente, lo cual en un futuro puede tener implicaciones terapéuticas importantes ${ }^{(12,13)}$.

Este estudio presenta datos que permiten conocer la distribución y el comportamiento de las lesiones selares en nuestro medio, lo cual es útil en la práctica clínica para el diagnóstico diferencial y decisiones terapéuticas. Se requieren más estudios con datos de seguimiento prospectivo y que integren variantes clínicas y radiológicas para un conocimiento más profundo de la patología selar en nuestro medio.

\section{Conclusión}

La distribución de los hallazgos histopatológicos de la región selar en esta serie es similar a lo reportado en la literatu- 
ra, llamando la atención un predominio del craneofaringioma como la segunda lesión más frecuente después de los adenomas hipofisarios. Se requiere una mayor uniformidad y difusión en los criterios histopatológicos de la clasificación de los adenomas para tener una interpretación clínica más apropiada y un uso óptimo de los recursos. Igualmente son necesarios nuevos estudios con seguimiento prospectivo y que integren datos clínicos y radiológicos para comprender mejor el comportamiento de estas lesiones en nuestro medio.

\section{Declaración de fuentes de financiación y posibles conflic- tos de interés}

No requirió financiación

No presentamos conflictos de interés

\section{Referencias}

1. McNeill KA. Epidemiology of Brain Tumors. Neurol Clin. 2016;34(4):98198. http://doi.org/10.1016/j.ncl.2016.06.014

2. Ostrom QT, Gittleman H, Fulop J, Liu M, Blanda R, Kromer C, et al. CBTRUS Statistical Report: Primary Brain and Central Nervous System Tumors Diagnosed in the United States in 2008-2012. Neuro Oncol. 2015;17:iv1-iv62. http://doi.org/10.1093/neuonc/nov189

3. Al-Futaisi A, Saif AY, Al-Zakwani I, Al-Qassabi S, Al-Riyami S, Wali Y. Clinical and epidemiological characteristics of pituitary tumours using a web-based pituitary tumour registry in Oman. Sultan Qaboos Univ Med J. 2007;7(1):25-30.

4. Arafah BM, Nasrallah MP. Pituitary tumors: Pathophysiology, clinical manifestations and management. Endocr Relat Cancer. 2001;8(4):287-305. http://doi.org/10.1677/erc.0.0080287

5. Ezzat S, Asa SL, Couldwell WT, Barr CE, Dodge WE, Vance ML, et al. The prevalence of pituitary adenomas: A systematic review. Cancer. 2004;101(3):613-9. http://doi.org/10.1002/cncr.20412

6. Lüdecke DK, Buchfelder M, Fahlbusch R, Quabbe HJ, Petersenn S, Saeger W. Pathohistological classification of pituitary tumors: 10 years of experience with the German Pituitary Tumor Registry. Eur J Endocrinol. 2007;156(2):203-16. http://doi.org/10.1530/eje.1.02326

7. Freda P, Post K. Differential Diagnosis of Sellar Lesions. Pituitary. 1999;28(1):81-117. http://doi.org/ 10.1016/S0889-8529(05)70058-X
8. Wade AN, Baccon J, Grady MS, et al. Clinically silent somatotroph adenomas are common. Eur J Endocrinol 2011;165(1):39-44. http://doi.org/ 10.1530/EJE-11-0216

9. Trouillas J, Roy P, Sturm N, Dantony E, Cortet-Rudelli C, Viennet G, et al. A new prognostic clinicopathological classification of pituitary adenomas: A multicentric case-control study of 410 patients with 8 years post-operative follow-up. Acta Neuropathol. 2013;126(1):123-35. http://doi.org/ 10.1007/s00401-013-1084-y

10. Kiseljak-Vassiliades K, Carlson NE, Borges MT, Kleinschmidt-DeMasters BK, Lillehei KO, Kerr JM, et al. Growth hormone tumor histological subtypes predict response to surgical and medical therapy. Endocrine. 2015;49(1):23141. http://doi.org/ 10.1007/s12020-014-0383-y

11. Lopes MBS. The 2017 World Health Organization classification of tumors of the pituitary gland: a summary. Acta Neuropathol. 2017;134(4):521-35. http://doi.org/ 10.1007/s00401-017-1769-8

12. Müller HL, Merchant TE, Puget S, Martinez-Barbera JP. New outlook on the diagnosis, treatment and follow-up of childhood-onset craniopharyngioma. Nat Rev Endocrinol. 2017;13(5):299-312. http://doi.org/ 10.1038/nrendo.2016.217

13. Müller HL. Craniopharyngioma. Endocr Rev. 2014;35(3):513-43. http:// doi.org/ doi.org/10.1210/er.2013-1115 